\title{
Radiographic and Arthroscopic Assessment of DRUJ Instability Due to Foveal Avulsion of the Radioulnar Ligament in Distal Radius Fractures
}

\author{
Toshiyasu Nakamura, MD, PhD ${ }^{1}$ Takuji Iwamoto, MD, $\mathrm{PhD}^{1} \quad$ Noboru Matsumura, MD, $\mathrm{PhD}^{1}$ \\ Kazuki Sato, MD, $\mathrm{PhD}^{1}$ Yoshiaki Toyama, MD, $\mathrm{PhD}^{1}$ \\ ${ }^{1}$ Department of Orthopaedic Surgery, School of Medicine, Keio \\ University, Tokyo, Japan \\ J Wrist Surg 2014;3:12-17. \\ Address for correspondence Toshiyasu Nakamura, MD, PhD., \\ Department of Orthopaedic Surgery, School of Medicine, Keio \\ University, 35 Shinanomachi, Shinjuku-ku, Tokyo 160-8582, Japan \\ (e-mail: toshiyasu@ae.em-net.ne.jp).
}

\begin{abstract}
Keywords

- distal radius fracture

- radial translation

- triangular fibrocartilage complex

- radioulnar ligament
\end{abstract}

Background As the triangular fibrocartilage complex (TFCC) anchors the distal radius to the ulna via the radioulnar ligament (RUL), a severely displaced distal fragment of the radius may be associated with a foveal avulsion of the TFCC. The purpose of this retrospective study was to assess, radiographically and arthroscopically, the relationship between displacement of the radius, the ulnar styloid, and avulsion of the RUL resulting in distal radioulnar joint (DRUJ) instability.

Materials and Methods Twenty-nine wrists of 29 patients with intra- and extra-articular distal radius fractures/malunion who underwent reduction or a corrective osteotomy of the displaced/malunited fracture, and/or wrist arthroscopy, were assessed radiographically and arthroscopically. Radial translation, radial inclination, radial shortening, volar or dorsal tilt, and the presence of an ulnar styloid fracture with more than $4 \mathrm{~mm}$ of displacement were measured from the initial films. Radiocarpal arthroscopy was used to assess peripheral lesions of the TFCC, while DRUJ arthroscopy was used to assess the foveal attachment. The relationship between displacement of the distal radius or the ulnar styloid fracture and the TFCC injury, including avulsion of the RUL, was recorded.

Results Univariate analysis revealed that increased radial translation, decreased radial inclination, increased radial shortening, and an ulnar styloid fragment radially displaced by more than $4 \mathrm{~mm}$ were significant predictors of RUL avulsion at the fovea. Volar or dorsal tilt of the radius and ulnar variance did not correlate with RUL avulsion or TFCC injuries. Multiple logistic regression analysis revealed that radial translation was an independent risk factor of foveal avulsion of the RUL.

Conclusions Increased radial translation and radial shortening and decreased radial inclination of the distal fragment can be associated with a foveal avulsion of the RUL. Radial translation can be an independent risk factor of foveal avulsion of the RUL.
Distal radius fractures are the most common injury of the upper extremity. The recent development of locking implants enables accurate restoration of the bony anatomy. ${ }^{1,2}$ Soft tissue injuries associated with a distal radius fracture may, however, result in residual wrist pain, decreased grip strength, and a restriction of wrist range of motion
(ROM). ${ }^{3,4}$ Tears of the triangular fibrocartilage complex (TFCC), scapholunate ligament, and lunotriquetral ligament are frequently associated with distal radius fractures. ${ }^{3-6}$

The TFCC is an important soft tissue stabilizer for the ulnar side of the wrist. $^{7}$ It consists of the triangular fibrocartilage (TFC); the radioulnar, ulnolunate, and ulnotriquetral ligaments;
Copyright @ 2014 by Thieme Medical Publishers, Inc., 333 Seventh Avenue, New York, NY 10001, USA. Tel: +1(212) 584-4662.
DOI http://dx.doi.org/ 10.1055/s-0033-1364175. ISSN 2163-3916. 
and the meniscus homologue and sheath floor of the extensor carpi ulnaris (ECU). The TFCC stabilizes the ulnocarpal joint and the distal radioulnar joint (DRUJ), distributes and absorbs load between the ulna and ulnar carpus, and allows smooth wrist motion and forearm rotation. ${ }^{7,8}$ The TFCC attaches distally to the lunate and triquetrum with the ulnolunate and ulnotriquetral ligaments and meniscus homologue and proximally to the ulna, via the fovea fibers. ${ }^{8,9}$

The fracture pattern of the distal radius, the magnitude of fracture displacement, and the presence of fracture of the ulnar styloid base correlate with TFCC injuries. ${ }^{10-12}$ Fujitani et $\mathrm{al}^{13}$ recently described that radial translation ratio (DRUJ gap) and sagittal translation ratio of the fracture site were significant predictors of DRUJ instability, as assessed by the DRUJ ballottement test, ${ }^{14}$ which can be an indirect and somewhat inaccurate technique for the diagnosis of DRUJ instability. DRUJ arthroscopy provides the most accurate diagnosis of a fovea tear through direct visualization and probing of the fovea insertion of the RUL. ${ }^{15}$

In the present study, we examined the radiographic parameters and performed DRUJ arthroscopy in a series of consecutive cases of distal radius fractures/malunion to define the radiographic predictors that are associated with a foveal avulsion of the RUL. A foveal avulsion of the TFCC was diagnosed by DRUJ arthroscopy.

\section{Materials and Methods}

Since 2008, 29 wrists of 29 patients with an intra- and extra$\operatorname{articular}$ (AO type A and B) distal radius fracture or malunited extra-articular fracture underwent an open reduction or a corrective osteotomy of the displaced/malunited fracture and wrist arthroscopy in our hospital. There were 8 male and 21 female patients with an average age of 49.8 years (range 13-79). The right wrist was affected in 19 patients and the left in 10. Twenty-four patients had an acute displaced fracture of the distal radius, and five patients had a malunion. Twenty patients with a displaced distal radius fracture underwent an open reduction and internal fixation (ORIF), with a volar locking plate. Cast immobilization was used for four patients who had a stable distal radius fracture but who underwent wrist arthroscopy only of persistent ulnar-sided wrist pain after the fracture healed. A displaced distal radius fracture was defined as at least one of $(1)$ dorsal tilt of greater than $19^{\circ}$, (2) volar tilt of greater than $19^{\circ}$, and (3) shortening of the radius equal and more than $5 \mathrm{~mm}$, or combination of those. Five patients with a dorsally malunited distal radius underwent a corrective osteotomy using volar locking plates. After plate fixation, DRUJ stability was examined using the DRUJ ballottement test. Wrist arthroscopy was performed to examine the TFCC condition in 20 patients at 6 months after the treatment for the distal radius (at the time of plate removal) who complained of residual ulnar-side wrist pain even at the time of plate removal. In five patients, radiocarpal and DRUJ arthroscopy was performed at the time of plate fixation because of persistent DRUJ instability. We excluded skeletally immature patients and patients with past ipsilateral injuries of the wrist. We also excluded comminuted intra-articular fracture of the radius (AO type $\mathrm{C}$ ) and malunion cases without initial radiographs at the time of distal radius fracture. The institutional review board of our hospital approved this retrospective analysis (20130147).

Standard posteroanterior (PA) and lateral radiographs were taken initially prior to any reduction of the distal radius fracture. The radial translation, radial inclination, degree of radial shortening, and ulnar variance were measured on the $\mathrm{PA}$ radiographs, and the volar tilt was measured on the lateral radiographs ( $\mathbf{- F i g . 1}$ ). Radial translation was defined as the maximum gap between the radial margin of the distal fragment and the radial margin of the shaft of the proximal radius. The radial inclination was the angle between a line perpendicular to the central axis of the radius and a line connecting the radial and ulnar limits of the articular surface of the distal radius. Radial shortening was an average shortening of the radial and ulnar cortex of the radius. Ulnar variance was defined as the difference in height between a perpendicular line drawn through the proximal margin of the sigmoid notch of the and the distal cortical rim of the ulnar head. ${ }^{16,17}$ Radial displacement of the ulnar styloid fracture was measured. The volar tilt was the angle between a line perpendicular to the central axis of the radius shaft and a line

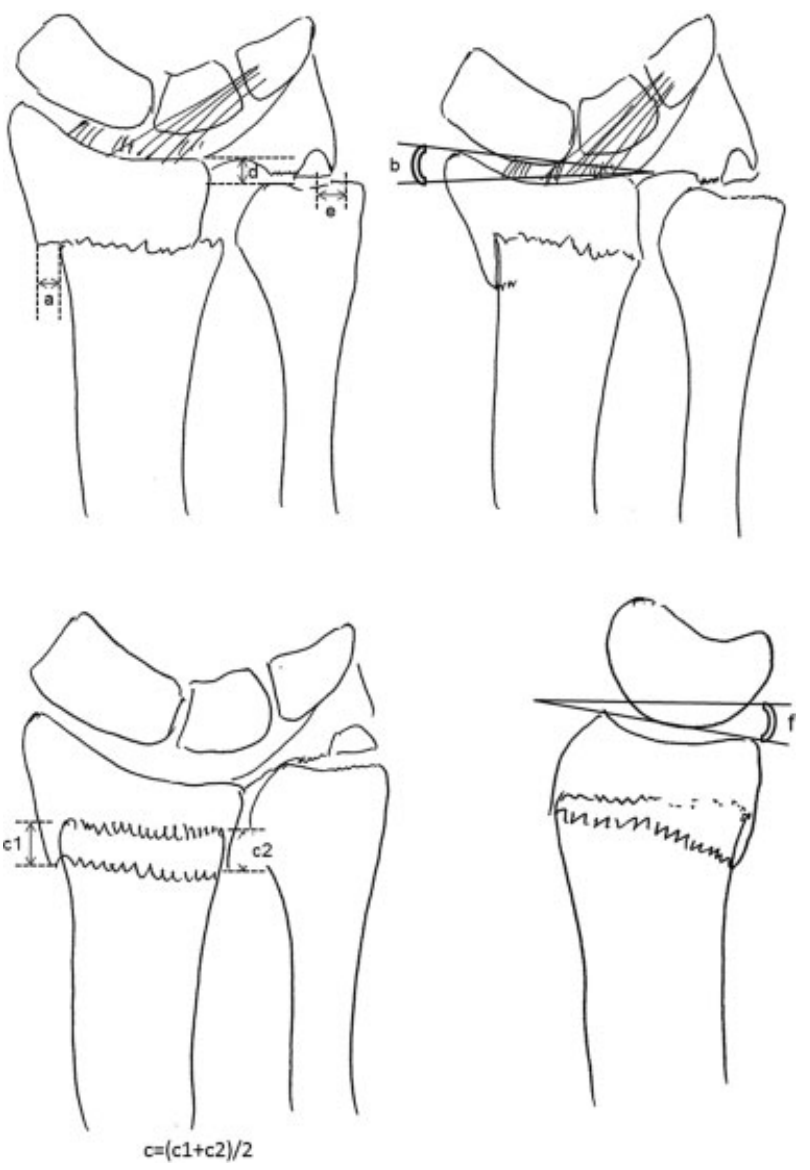

Fig. 1 Illustration of radiographic parameters on radial translation (a), radial inclination (b), radial shortening, which was calculated as average shortening of radial shaft and ulnar shaft (c), ulnar variance (d), and displacement of the ulnar styloid fragment (e) on PA view and volar tilt (f) on lateral view. 


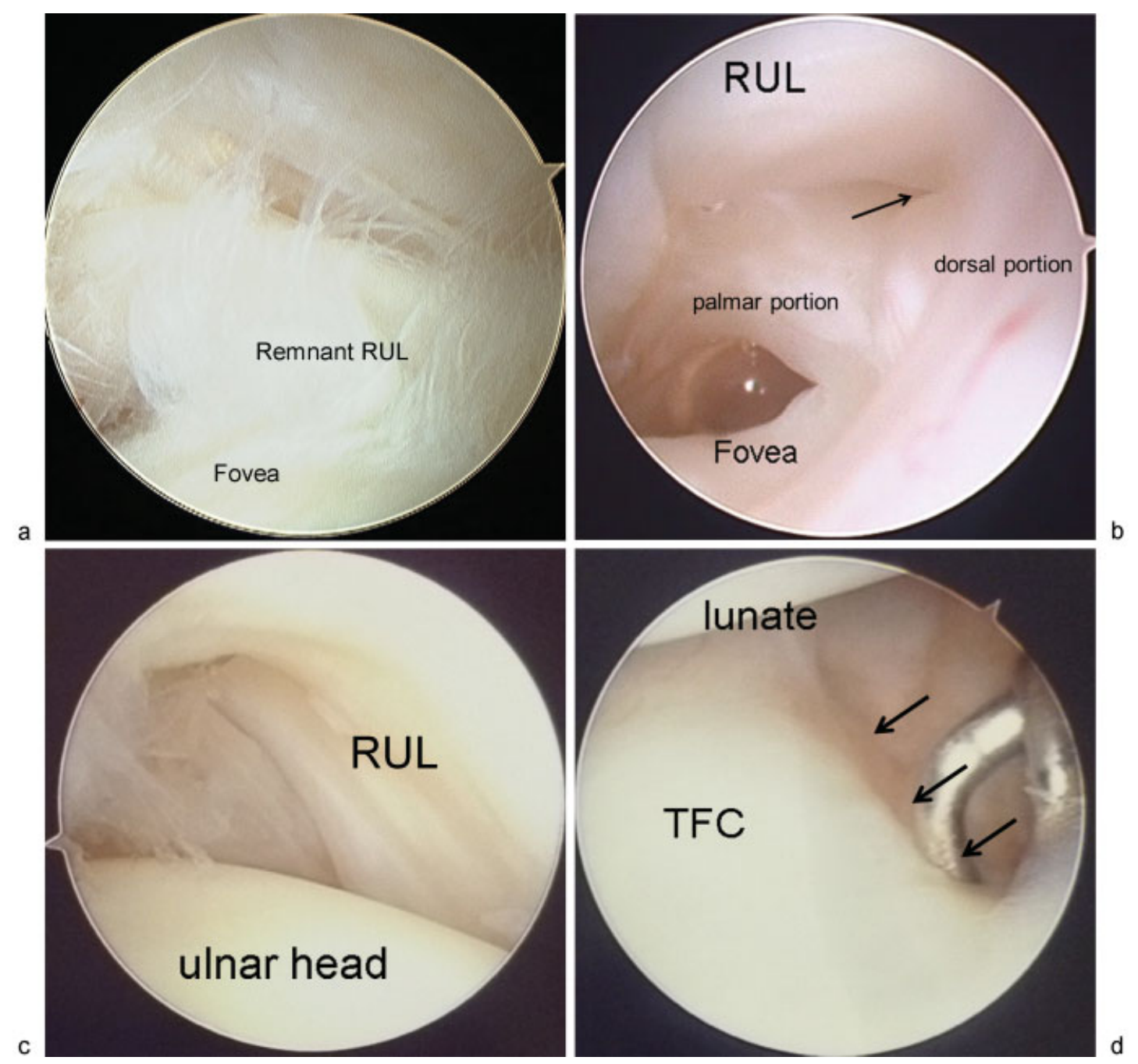

Fig. 2 (a) There were 12 wrists that had a complete foveal avulsion of the RUL at the fovea. (b) One wrist had partial avulsion of the RUL (arrow) at the fovea. (c) Sixteen wrists were found to have intact RUL in the distal radius fractures via DRUJ arthroscopy, (d) Radiocarpal arthroscopy also revealed a peripheral tear of the triangular fibrocartilage from the capsule (Palmer 1B tear; arrows) in three wrists.

connecting the dorsal and palmar margin of the articular surface of the distal radius on the lateral radiographs. The dorsal tilt was defined as a negative value of the volar tilt.

Univariate association analysis between the potential predictors on radiographs and the outcome variable, which was the presence or absence of total or partial avulsion of the RUL at the fovea, was performed using the nonparametric MannWhitney U-test or chi-square test with Fisher's direct extract for categorical variables. Multiple logistic regression analysis (Sigma Plot, ver. 12.0, Systat Software, Inc., San Jose, CA, USA) was also performed to assess the relationship between radiographic parameters as categorical explanatory variables and the presence or absence of total or partial avulsion of the RUL at the fovea as a single categorical response variable. Values of $p<0.05$ were considered statistically significant.

\section{Results}

Twelve of 29 patients (41\%) were found to have a complete avulsion of the RUL at the time of DRUJ arthroscopy
(-Fig. 2a), while one patient had a partial avulsion of the RUL (3\%) (-Fig. 2b). An open repair of the RUL was performed in 10 wrists, a combination of an open repair of the RUL and ulnar shortening was done in one patient, and a reconstruction of the TFCC using a half-slip of the ECU tendon was performed in one patient for a complete foveal avulsion of the RUL. An ulnar shortening procedure was done in the patient with a partial foveal avulsion. In the other 16 wrists, the RUL was intact (-Fig. 2c). Three patients had a peripheral Palmer 1B TFCC tear that was treated with an arthroscopic capsular repair (-Fig. 2d). Seven patients were noted to have a degenerative tear (Palmer class 2) of the TFCC associated with a positive ulnar variance and were treated with an ulnar shortening osteotomy at the time the plate in the radius was removed. An arthroscopic synovectomy was performed in six patients with synovial proliferation but no obvious TFCC lesion.

Radial translation ( - Fig. 3a) was found in eight wrists with an average of $7.6 \mathrm{~mm}$ of displacement, while 21 patients had no radial translation. Thirteen wrists with RUL avulsion had 


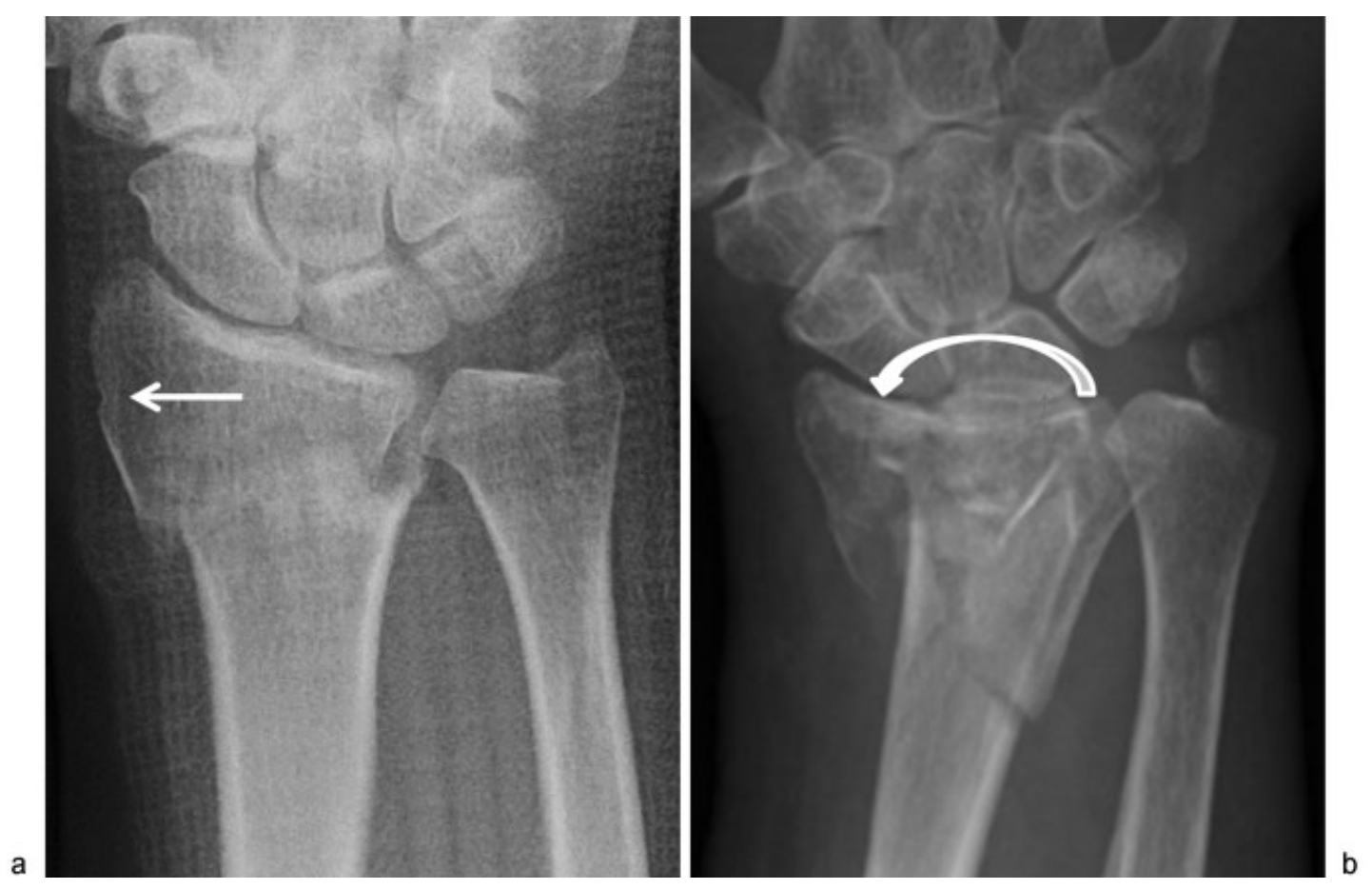

Fig. 3 Radiographs of (a) radially displaced (white arrow) fragment and (b) severely decreased radial inclination (white round arrow).

average $4.7 \mathrm{~mm}$ of radial translation, while average $0.1 \mathrm{~mm}$ of radial translation was noted in the 16 wrists with intact RUL. The radial inclination ranged from $-9^{\circ}$ to $28^{\circ}$ with an average of $16.7^{\circ}$ totally, indicating average $11.5^{\circ}$ in 13 wrists with RUL avulsion (-Fig. 3b) and average $21.0^{\circ}$ in 16 wrists without RUL avulsion. Radial shortening averaged $4.86 \mathrm{~mm}$ (range: 0 to $15.6 \mathrm{~mm}$ ). In the wrists with RUL avulsion the average radial shortening was $6.7 \mathrm{~mm}$, compared to radial shortening of $3.4 \mathrm{~mm}$ in the wrists with an intact RUL. The average ulnar variance was $+2.9 \mathrm{~mm}$ (range $0-7.2 \mathrm{~mm}$ ). In the wrists with an RUL avulsion the average ulnar variance was $+3.0 \mathrm{~mm}$, and the wrists with an intact RUL had an average of $+2.9 \mathrm{~mm}$. The volar tilt ranged from $-35^{\circ}$ to $35^{\circ}$ (average $-8.4^{\circ}$ ); $-12.1^{\circ}$ in the wrists with an avulsed as compared with $5.4^{\circ}$ in the wrists with an intact RUL. Radial translation of the ulnar styloid fragment of more than $4 \mathrm{~mm}$ was found in 7 wrists with a RUL avulsion. Twenty-two wrists did not have any displacement of the ulnar styloid fragment: six of these wrists had a RUL avulsion, whereas 16 wrists had an intact RUL.

All eight wrists with radial translation had either a complete (seven wrists) or partial (one wrist) avulsion of the RUL at the fovea. Among the 21 wrists that had no radial translation, complete avulsion of the RUL at the fovea was found in five wrists, while 16 wrists had no RUL avulsion. The radial inclination, radial shortening, ulnar variance, and volar tilt of the eight wrists that had radial translation were $7.2^{\circ}, 5.6 \mathrm{~mm}$, $3.78 \mathrm{~mm}$, and $-11.6^{\circ}$, respectively, while those of the wrists that had no radial translation were $20.4^{\circ}, 4.6 \mathrm{~mm}, 2.6 \mathrm{~mm}$, and $-7.2^{\circ}$, respectively.

In the univariate analyses, an increased radial translation $(p=0.001)$, decreased radial inclination $(p=0.020)$, in- creased radial shortening $(p=0.007)$, and radial displacement of an ulnar styloid fragment of more than $4 \mathrm{~mm}$ ( $p=0.001$ ) were significant predictors of a foveal avulsion of the RUL, which resulted in DRUJ instability. Ulnar variance and volar or dorsal tilt were not significant predictors for foveal avulsion (-Table $\mathbf{1}$ ).

Only radial translation of the distal fragment of the radius was correlated with an increased incidence of a foveal avulsion of the RUL in multiple logistic regression analysis $(p=0.0003)$. Age, gender, decreased radial inclination, increased radial shortening, ulnar variance, and a displaced ulnar styloid fragment were not significant variables $(p=0.94-0.99)$.

\section{Discussion}

A TFCC injury is frequently associated with a distal radius fracture. The incidence ranges from $39 \%$ to $84 \%{ }^{3,6,10,13}$ The incidence of a foveal RUL tear diagnosed by manual testing, such as the ballottement test or radiocarpal arthroscopy findings in distal radius fractures, ranged from 7 to $33 \%^{3,6,12,13,18}$ In this study using DRUJ arthroscopy, an associated RUL avulsion was seen in 13 of 29 patients, or $45 \%$. A peripheral Palmer 1B tear was seen in 3 of 29 patients, or $10 \%$. Thus, the overall incidence of a traumatic TFCC injury associated with a distal radius fracture was $55 \%$. The previous studies used an indirect technique to diagnose the foveal avulsion of the TFCC via radiocarpal arthroscopy based on loss of the TFCC tension or a positive hook test (whereby the entire TFCC can be pulled proximally and radially with a hook probe). ${ }^{13,14}$ These indirect methods, however, may underestimate incidence of an RUL foveal avulsion in distal radius 
Table 1 Univariate analyses for predictors of radiographic parameters for RUL avulsion

\begin{tabular}{|l|l|l|l|}
\hline & Cases $(\boldsymbol{n}=\mathbf{1 3})$ & Control $(\boldsymbol{n}=\mathbf{1 6})$ & $\boldsymbol{p}$-Value \\
\hline Age & $45 \pm 23$ & $53 \pm 20$ & 0.28 \\
\hline Gender & & & 0.40 \\
\hline Female & 8 & 13 & \\
\hline Male & 5 & 3 & \\
\hline Radial translation $(\mathrm{mm})$ & $4.3 \pm 4.7$ & $0.2 \pm 1.2$ & 0.001 \\
\hline Radial inclination $\left(^{\circ}\right)$ & $11.5 \pm 11.1$ & $21.0 \pm 3.5$ & 0.020 \\
\hline Radial shortening $(\mathrm{mm})$ & $6.7 \pm 4.0$ & $3.4 \pm 2.3$ & 0.007 \\
\hline Ulnar variance $(\mathrm{mm})$ & $3.0 \pm 2.8$ & $2.9 \pm 2.4$ & 0.98 \\
\hline Volar tilt $\left(^{\circ}\right)$ & $-12.1 \pm 15.7$ & $-5.4 \pm 19.3$ & 0.34 \\
\hline Ulnar styloid fracture $(>4 \mathrm{~mm}$ of radial displacement) & $7(53 \%)$ & $0(0 \%)$ & 0.001 \\
\hline
\end{tabular}

fractures. DRUJ arthroscopy is more accurate because of the direct visualization of the foveal attachment. ${ }^{15}$

As the TFCC is the primary stabilizer of the DRUJ, avulsion of the TFCC at the fovea results in severe DRUJ instability. ${ }^{19}$ Fracture pattern and magnitude of displacement of the distal fragment in distal radius fracture are considered potential risk factors for DRUJ instability. ${ }^{10,11,13}$ Richards et al ${ }^{10}$ reported that the incidence of a TFCC injury following a distal radius fracture was related to the magnitude of radial shortening and dorsal angulation. Presence or greater displacement of ulnar styloid fracture in distal radius fracture was also a predicting factor for DRUJ instability, ${ }^{11}$ whereas recent prospective studies denied the influence of ulnar styloid fracture on TFCC injury ${ }^{12}$ or on the clinical outcome of surgical treatment for distal radius fracture, ${ }^{20,21}$ although they did not use wrist arthroscopy for diagnosis of TFCC injury.

In the present study, radial translation $(p=0.001)$, decreased radial inclination $(p=0.020)$, and increased radial shortening ( $p=0.007)$ were significantly associated with a foveal avulsion of the RUL, which was confirmed by DRUJ arthroscopy. The distal fragment of the radius and carpus can displace when the TFCC is no longer anchored to the fovea. When the TFCC is anchored to the fovea of the ulna through the RUL, the distal fragment cannot translocate radially and rotate proximally to the same degree. Ulnar variance and volar or dorsal tilt were not significant predictors for a foveal avulsion of the RUL.

Multiple logistic regression analysis revealed that only radial translation indicated independent risk for foveal avulsion of the RUL $(p=0.0003)$. This result strongly suggests that when clinicians treat a distal radius fracture with radial displacement, the DRUJ stability should be assessed clinically after internal fixation of the distal radius fracture, and if there is any suspicion, the foveal fibers should be assessed by either DRUJ arthroscopy or an arthrotomy to rule out a foveal avulsion of the RUL. .

Although radial displacement of an ulnar styloid fragment of more than $4 \mathrm{~mm}$ was also a significant contributor
( $p=0.001)$ of a foveal avulsion of the RUL in the univariate statistical analysis, it did not prove to be statistically significant in multiple logistic regression analysis. This may suggest that radial displacement of an ulnar styloid fragment of more than $4 \mathrm{~mm}$ was a related (confounding) parameter of radial translation of the radius. When the RUL is attached to the fovea, radial displacement of the ulnar styloid fragment may be minimized.

The radius connects to the scaphoid and lunate with strong extrinsic volar and dorsal radiocarpal ligaments such as the radioscaphocapitate, long and short radiolunate, and dorsal radiocarpal ligaments. During forearm pronosupination, both the radius and carpus rotate over the ulnar head. ${ }^{7}$ With an extra-articular fracture, the distal fragment is usually supinated and displaced with the proximal carpal row either in a dorsal, volar, or radial direction. In this situation, the TFCC is a key structure in preventing radial translation of the distal fragment of the radius, because the TFCC directly connects the distal radius to the ulnar fovea and base of the ulnar styloid via the RUL or indirectly connects those via the ulnocarpal ligaments, proximal carpus, and extrinsic radiocarpal ligaments.

\section{Conflict of Interest}

None

\section{References}

1 Grewal R, MacDermid JC, King GJW, Faber KJ. Open reduction internal fixation versus percutaneous pinning with external fixation of distal radius fractures: a prospective, randomized clinical trial. J Hand Surg Am 2011;36(12):1899-1906

2 Osada D, Kamei S, Masuzaki K, Takai M, Kameda M, Tamai K. Prospective study of distal radius fractures treated with a volar locking plate system. J Hand Surg Am 2008;33(5):691-700

3 Geissler WB, Freeland AE, Savoie FH, McIntyre LW, Whipple TL. Intracarpal soft-tissue lesions associated with an intra-articular fracture of the distal end of the radius. J Bone Joint Surg Am 1996; 78(3):357-365 
4 Geissler WB, Fernandez DL, Lamey DM. Distal radioulnar joint injuries associated with fractures of the distal radius. Clin Orthop Relat Res 1996;336(327):135-146

5 Lindau T, Hagberg L, Adlercreutz C, Jonsson K, Aspenberg P. Distal radioulnar instability is an independent worsening factor in distal radial fractures. Clin Orthop Relat Res 2000;376(376):229-235

6 Lindau T, Adlercreutz C, Aspenberg P. Peripheral tears of the triangular fibrocartilage complex cause distal radioulnar joint instability after distal radial fractures. J Hand Surg Am 2000; 25(3):464-468

7 Nakamura T, Yabe Y, Horiuchi Y. Functional anatomy of the triangular fibrocartilage complex. J Hand Surg Eur Vol 1996; 21(5):581-586

8 Nakamura T, Yabe Y. Histological anatomy of the triangular fibrocartilage complex of the human wrist. Ann Anat 2000; 182(6):567-572

9 Nakamura T, Takayama S, Horiuchi Y, Yabe Y. Origins and insertions of the triangular fibrocartilage complex: a histological study. J Hand Surg [Br] 2001;26(5):446-454

10 Richards RS, Bennett JD, Roth JH, Milne K Jr. Arthroscopic diagnosis of intra-articular soft tissue injuries associated with distal radial fractures. J Hand Surg Am 1997;22(5):772-776

11 May MM, Lawton JN, Blazar PE. Ulnar styloid fractures associated with distal radius fractures: incidence and implications for distal radioulnar joint instability. J Hand Surg Am 2002;27(6): 965-971

12 Bombaci H, Polat A, Deniz G, Akinci O. The value of plain X-rays in predicting TFCC injury after distal radius fractures. J Hand Surg Eur Vol 2008;33(3):322-326
13 Fujitani R, Omokawa S, Akahane M, Iida A, Ono H, Tanaka Y. Predictors of distal radioulnar joint instability in distal radius fractures. J Hand Surg Am 2011;36(12):1919-1925

14 Moriya T, Aoki M, Iba K, Ozasa Y, Wada T, Yamashita T. Effect of triangular ligament tears on distal radioulnar joint instability and evaluation of three clinical tests: a biomechanical study. J Hand Surg Eur Vol 2009;34(2):219-223

15 Nakamura T. DRUJ instability: arthroscopic repair of the detached TFCC to the fovea of the ulna. In: Del Pinal F, Mathoulin C, Nakamura T, eds. Arthroscopic management of ulnar pain. Berlin, Germany: Springer-Verlag; 2012:137-146

16 Palmer AK, Glisson RR, Werner FW. Ulnar variance determination. J Hand Surg Am 1982;7(4):376-379

17 Metz VM, Gilula LA. Imaging techniques for distal radius fractures and related injuries. Orthop Clin North Am 1993;24(2):217-228

18 Shih JT, Lee HM, Hou YT, Tan CM. Arthroscopically-assisted reduction of intra-articular fractures and soft tissue management of distal radius. Hand Surg 2001;6(2):127-135

19 Nakamura T, Sato K, Okazaki M, Toyama Y, Ikegami H. Repair of foveal detachment of the triangular fibrocartilage complex: open and arthroscopic transosseous techniques. Hand Clin 2011;27(3): 281-290

$20 \mathrm{Kim}$ JK, Koh YD, Do NH. Should an ulnar styloid fracture be fixed following volar plate fixation of a distal radial fracture? J Bone Joint Surg Am 2010;92(1):1-6

21 Zenke Y, Sakai A, Oshige T, Moritani S, Nakamura T. The effect of an associated ulnar styloid fracture on the outcome after fixation of a fracture of the distal radius. J Bone Joint Surg $\mathrm{Br}$ 2009;91(1): 102-107 